Research paper

\title{
Safety of coronary CT angiography and functional testing for stable chest pain in the PROMISE trial: A randomized comparison of test complications, incidental findings, and radiation dose
}

\author{
Michael T. Lu ${ }^{\text {a, }}$, Pamela S. Douglas ${ }^{\text {b }}$, James E. Udelson ${ }^{\text {c }}$, Elizabeth Adami a, \\ Brian B. Ghoshhajra ${ }^{a}$, Michael H. Picard ${ }^{a}$, Rhonda Roberts ${ }^{b}$, Kerry L. Lee ${ }^{b}$, \\ Andrew J. Einstein ${ }^{\mathrm{d}}$, Daniel B. Mark ${ }^{\mathrm{b}}$, Eric J. Velazquez ${ }^{\mathrm{b}}$, William Carter ${ }^{\mathrm{e}}$, \\ Michael Ridner ${ }^{\mathrm{f}}$, Hussein R. Al-Khalidi ${ }^{\mathrm{b}}$, Udo Hoffmann ${ }^{\mathrm{a}}$ \\ ${ }^{a}$ Massachusetts General Hospital and Harvard Medical School, Boston, MA, USA \\ b Duke Clinical Research Institute, Duke University School of Medicine, Durham, NC, USA \\ ' Tufts Medical School, Boston, MA, USA \\ ${ }^{\mathrm{d}}$ Columbia University Medical Center and New York-Presbyterian Hospital, New York, NY, USA \\ e Charleston Area Medical Center, Charleston, WV, USA \\ ${ }^{\mathrm{f}}$ University of Alabama School of Medicine, Huntsville, AL, USA
}

\section{A R T I C L E I N F O}

\section{Article history:}

Received 6 June 2017

Received in revised form

25 July 2017

Accepted 12 August 2017

Available online $\mathrm{xxx}$

\section{Keywords:}

Coronary computed tomography

angiography

Functional testing

Chest pain

Coronary artery disease

\begin{abstract}
A B S T R A C T
Background: Coronary computed tomography angiography (CTA) and functional testing strategies for stable chest pain yield similar outcomes; one aspect that may guide test choice is safety.

Methods: We compared test safety (test complications, incidental findings, and effective radiation dose) between CTA and functional testing as-tested in PROMISE (PROspective Multicenter Imaging Study for Evaluation of Chest Pain). In the subgroup whose physicians intended nuclear stress over other functional tests if randomized to the functional arm, we compared radiation dose of CTA versus nuclear stress and identified characteristics associated with dose.

Results: Of 9470 patients, none had major and $<1 \%$ had minor complications (CTA: $0.8 \%$ [37/4633] vs. functional: 0.6\% [27/4837]). CTA identified more incidental findings (11.6\% [539/4633] vs. 0.7\% [34/4837], $\mathrm{p}<0.001)$, most commonly pulmonary nodules $(9.4 \%, 437 / 4633)$. CTA had similar 90 -day cumulative radiation dose to functional testing. However, in the subgroup whose physicians intended nuclear stress (CTA 3147; nuclear 3203), CTA had lower median index test ( 8.8 vs. $12.6 \mathrm{mSv}, \mathrm{p}<0.001$ ) and 90-day cumulative (11.6 vs. $13.1 \mathrm{mSv}, \mathrm{p}<0.001$ ) dose, independent of patient characteristics. The lowest nuclear doses employed 1-day Tc-99m protocols (12.2 mSv). The lowest CTA doses were at sites performing $\geq 500 \mathrm{CTAs} /$ year $(6.9 \mathrm{mSv})$ and with advanced (latest available) CT scanners (5.5 mSv).

Conclusion: Complications were negligibly rare for both CTA and functional testing. CTA detects more incidental findings. Compared to nuclear stress testing, CTA's lower radiation dose, independent of patient characteristics, makes it an attractive test choice. Radiation dose varies with imaging protocol, indicating opportunities to further reduce dose. (ClinicalTrials.gov number, NCT01174550).
\end{abstract}

(c) 2017 Society of Cardiovascular Computed Tomography. Published by Elsevier Inc. All rights reserved.

* Corresponding author. Massachusetts General Hospital, 165 Cambridge St, Suite 400, Boston, MA 02114, USA.

E-mail addresses: mlu@mgh.harvard.edu (M.T. Lu), pamela.douglas@dm.duke.edu (P.S. Douglas), judelson@tuftsmedicalcenter.org (J.E. Udelson), eadami@partners.org

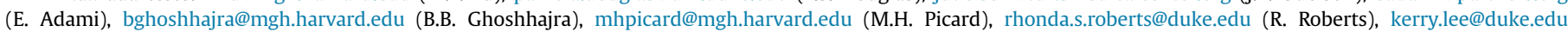

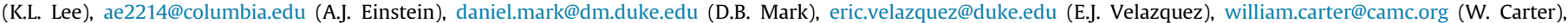
mridner@theheartcenter.md (M. Ridner), hussein.al-khalidi@duke.edu (H.R. Al-Khalidi), uhoffmann@partners.org (U. Hoffmann). 

Abbreviations
CAD coronary artery disease
CT computed tomography
CTA CT angiography
ETT exercise tolerance testing

\section{Introduction}

Stable chest pain is one of the most common indications for noninvasive diagnostic testing in the United States, with over 4 million Americans undergoing outpatient testing for suspected coronary artery disease (CAD) annually. ${ }^{1}$ Most have a functional test (exercise tolerance testing [ETT], stress echocardiography, or nuclear stress testing). Recent randomized controlled trials have demonstrated that anatomic coronary computed tomography angiography (CTA) is a maturing diagnostic alternative with similar cardiovascular outcomes. ${ }^{2,3}$

One aspect that may contribute to test choice is test safety. Early reports of high radiation dose and frequent incidental findings have been barriers to the adoption of CTA. ${ }^{4-6}$ A randomized as-tested comparison of test safety between CTA and functional testing, with safety defined by test complications, incidental findings, and radiation dose, has not been performed. PROMISE (PROspective Multicenter Imaging Study for Evaluation of Chest Pain), a pragmatic multicenter randomized controlled trial of an initial anatomic (CTA) versus functional testing strategy for stable chest pain, presents an opportunity to compare the safety of CTA and functional testing. ${ }^{2}$ In PROMISE, anatomic CTA and functional testing strategies had similar rates of adverse cardiovascular events, test complications, and 90-day cumulative radiation dose; however, this as-randomized comparison included patients who were not tested or had the other test.

We therefore carried out an as-tested assessment of the safety of CTA versus functional testing in PROMISE based on test complications, incidental findings, and index test and 90-day cumulative radiation dose. Furthermore, in the subgroup of patients whose physicians prespecified nuclear stress testing as the intended functional test if the patient were randomly assigned to the functional testing group, we compared the radiation dose of CTA versus nuclear stress testing and identified patient and technical characteristics associated with dose.

\section{Methods}

\subsection{Population and study design}

This prespecified analysis was planned within the PROMISE trial; the design and results of the trial are detailed elsewhere. ${ }^{2,7}$ Briefly, after providing written informed consent, outpatients with stable chest pain and without known CAD were enrolled at 193 North American sites between July 2010 and September 2013. Subjects were randomly assigned to an initial anatomic (CTA) or functional (exercise or pharmacologic nuclear stress, stress echocardiography, or ETT per physician choice) testing strategy. Baseline characteristics including cardiovascular risk factors were collected at enrollment. Prior to randomization, treating physicians specified which functional test the patient should receive if he or she were randomized to the functional arm, ${ }^{7}$ with randomization stratified by this preference (i.e., those patients whose physicians preferred nuclear testing were randomized in a 1:1 ratio to CTA vs. functional testing). Subjects were followed for a median of 25 months for a composite of major adverse cardiovascular events including death, myocardial infarction, hospitalization for unstable angina, and major procedural complications as adjudicated by an independent clinical events committee.

In the first part of this as-tested analysis, test complications, potentially significant incidental findings, and 90-day cumulative radiation dose were compared between CTA and functional testing. The second part of the analysis focused on radiation dose in the subgroup of patients whose physician specified that they intended nuclear stress testing should the patient be randomized to the functional arm. In this subgroup, index test radiation dose and 90day cumulative radiation dose were compared between CTA and nuclear stress testing, again as tested. Patient, site experience, and technological factors associated with radiation dose were described.

All PROMISE subjects who had functional testing or CTA as their first test were included. Patients who had no test or who had invasive coronary angiography (ICA) as their first test were excluded. Computed tomography $(\mathrm{CT})$ protocols commonly include a non-contrast $\mathrm{CT}$ for assessment of coronary artery calcium score before contrast-enhanced CTA to assess coronary artery stenosis. Some sites chose not to proceed with CTA in patients with a high calcium score ${ }^{8}$; these participants were excluded as the radiation dose from calcium score CT alone is substantially lower than for CTA.

\subsection{Diagnostic testing}

CTA, ETT, stress echocardiography, and exercise or pharmacologic nuclear stress testing were performed by local physicians in accordance with professional society guidelines. Electrocardiography (ECG)-gated CTA was performed on single or dual source CT scanners with at least 64 slices. ${ }^{8,9}$ For nuclear stress testing, the imaging protocol and administered agent were recorded; for CTA, the CT scanner model and whether a prospective or retrospective ECG-triggered or gated protocol was used were recorded. "Advanced" CT scanners were defined as 64 + slice CT scanners that were each of the 4 largest vendors' newest model during the study period (GE Discovery CT750 HD, Philips Brilliance iCT, Siemens Definition Flash, Toshiba Aquilion ONE), with all other CT scanners considered "standard." At the beginning of the trial, sites completed surveys in which they reported their annual volume of nuclear stress and CTA testing as a measure of their experience.

\subsection{Test complications}

Prespecified test complications were collected by site personnel and considered up to $24 \mathrm{~h}$ after the randomized test. For both CTA and functional testing, major complications were defined as death, renal failure requiring dialysis, or anaphylaxis requiring emergency respiratory and/or circulatory support. For CTA, minor complications included mild intravenous contrast reactions, contrast extravasation, and adverse reactions to nitroglycerin or beta blockers administered during the examination. For functional testing, minor complications included hemodynamic instability or hypotension, arrhythmia, and adverse drug reactions. Hospitalizations related to test complications were also collected. A full list of test complications and their definitions is provided in the Supplementary Material.

\subsection{Incidental findings}

Site physicians reported prespecified potentially clinically significant incidental findings found on CTA, stress echocardiography, 
and nuclear stress testing. Potentially significant incidental findings on CTA included coronary anomalies, lung nodules, pulmonary embolism, pneumonia, aortic aneurysm, aortic dissection, and hiatal hernia, which could be a source for chest pain. For stress echocardiography these included moderate or large pericardial effusion or tamponade, moderate or severe aortic or mitral stenosis, moderate or severe aortic insufficiency, moderate or severe mitral regurgitation, moderate or severe pulmonary hypertension, hypertrophic obstructive cardiomyopathy, endocarditis, mitral prolapse, and aortic root aneurysm. For nuclear stress testing, these included radiotracer uptake within the lung, breast, or axilla.

\subsection{Estimated effective radiation dose}

Effective radiation dose was estimated in units of millisieverts $(\mathrm{mSv})$ using standard methods. ${ }^{4,10-12}$ For CTA, the estimated effective dose was calculated as the product of the dose length product (DLP) and a standard chest k-factor of $0.014 \mathrm{mSv} \mathrm{mGy}^{-1} \mathrm{~cm}^{-113}$. For nuclear stress imaging, the estimated simple effective dose was calculated as the product of the administered activity by the effective dose coefficient for that tracer and protocol. ${ }^{14,15}$ Cumulative 90-day effective radiation dose was defined as radiation dose due to the index test plus any additional cardiovascular testing or procedures performed within 90 days after randomization, including CTA, nuclear stress testing, ICA, and coronary interventions. The protocol for imputation of ICA, CTA, and nuclear stress testing radiation doses is described in the Supplementary Material.

\subsection{Statistical analysis}

Demographics, risk factors, and the composite outcome of major adverse cardiovascular events are presented as medians (interquartile range [IQR]) and means (standard deviations [SD]) for continuous variables; the frequency (percentage) of patients in each category are presented for nominal variables.

In the first part of the analysis, comparisons between the CT and functional testing groups were performed using the Wilcoxon rank sum test for continuous variables and Pearson's chi-square or Fisher exact tests for categorical variables. Diagnostic test complications and incidental findings were tabulated and compared between the patients having CT versus functional testing. Likewise, the 90-day cumulative effective radiation dose was tabulated and compared between the CT and functional testing groups.

The second part of the analysis focused on comparisons of radiation dose between $\mathrm{CT}$ and nuclear stress testing in the randomization strata of patients whose physicians intended to refer for nuclear stress testing if the patient were randomly assigned to the functional testing strategy. First the index test radiation dose in $\mathrm{mSv}$ of patients having nuclear stress versus CTA was compared using the Wilcoxon rank sum test. The percent of index test doses $\leq 9 \mathrm{mSv}$ was compared against the American Society of Nuclear Cardiology goal that $50 \%$ of nuclear stress tests have a radiation dose $\leq 9 \mathrm{mSv}{ }^{16}$ As a secondary outcome, 90-day cumulative radiation dose was also compared. Radiation dose was then summarized within specific prospectively chosen subgroups to assess whether patient-specific factors known to affect dose-including sex, age $\geq 65$ years, obesity (body mass index [BMI] $\geq 30 \mathrm{~kg} / \mathrm{m}^{2}$ ), and baseline resting heart rate at the enrollment physical examination $\geq 75$ beats per minute-changed the relationship between nuclear stress and CTA index test and 90-day cumulative radiation dose using the Wilcoxon rank sum test. For each of the above categories, a multivariable model was developed to compare CTA and nuclear stress testing radiation dose while controlling for the other patient-specific factors. Second, $\mathrm{R}^{2}$ values for each patient factor were then compared to assess the relative effect on the dose of CTA versus nuclear stress testing. Finally, radiation dose was then summarized based on site- and protocolspecific factors, including use of an advanced CT scanner, retrospective versus prospectively ECG-gated CTA protocols, nuclear stress test protocol and tracer, and site self-reported annual volume of nuclear stress and CTA testing.

Statistical analysis was performed using SAS (version 9.4) and JMP Pro (version 12) from the SAS Institute (Cary, North Carolina). All statistical testing was 2-sided using a level of significance of 0.05 .

\section{Results}

\subsection{Patient population, characteristics, and adverse cardiovascular events}

Of the 10,003 patients enrolled in PROMISE, 404 had no testing and 29 had ICA as a first test (Fig. 1A). Of the remaining 9570 patients, 4733 had CT and 4837 had functional testing. Of the 4733 who had CT, 4633 completed CTA while 100 had only a noncontrast calcium score CT. Of the 4837 having functional testing, 3263 had nuclear stress testing, 1083 had stress echocardiography, and 491 had ETT. Baseline characteristics and adverse cardiovascular events were similar between the groups having CTA and functional testing (Table 1).

\subsection{Test complications - CTA versus functional testing}

There were no major test complications (cardiac arrest, severe bronchospasm, anaphylaxis, renal failure requiring dialysis, or death) in either arm. Minor test complications were similarly rare for CTA $(0.8 \%, 37 / 4633)$ and functional testing $(0.6 \%, 27 / 4837$, $\mathrm{p}=0.15$, Table 2 ), with individual complications detailed in Table 3. For CTA, the most common minor complications were mild contrast reactions $(0.5 \%, 22 / 4633)$ and contrast extravasation at the site of the IV $(0.3 \%, 12 / 4633)$. For stress echocardiography, the most common complication was hypotension $(0.2 \%, 2 / 1083)$. For nuclear stress testing, the most common minor complications were hypotension $(0.2 \%, 6 / 3263)$, ventricular tachycardia $(0.2 \%, 5 / 3263)$, and dipyridamole/adenosine-related events $(0.2 \%, 5 / 3263)$. Overall, 5 patients in the functional arm (ETT $n=1$, stress echocardiography $\mathrm{n}=1$, nuclear stress testing $\mathrm{n}=3$ ) and none having CTA were hospitalized due to complications attributed to the index test.

\subsection{Incidental findings - CTA versus functional testing}

More patients had potentially significant incidental findings with CTA than with functional testing (11.6\%, $539 / 4633$ vs. $0.7 \%, 34$ / 4,837, p < 0.001) (Table 3). The most common CTA incidental finding was lung nodules $(9.4 \%, 437 / 4633)$. Other CTA findings with the potential to cause chest pain included coronary anomalies (1.5\%, 71/4633), pulmonary embolism $(0.1 \%, 4 / 4633)$, pneumonia $(0.2 \%, 9 / 4633)$, and aortic dissection $(0.2 \%, 8 / 4633)$. In all, $2.0 \%(93 /$ 4633 ) had one of the above findings potentially causing chest pain. In addition, 7.7\% (358/4633) had hiatal hernia and 1.9\% (89/4633) had aortic aneurysm, both of which in some instances can also be a source of chest pain. In the functional arm, abnormal breast uptake of radiotracer $(0.3 \%, 11 / 3263)$ on nuclear stress testing, and mitral regurgitation $(1.0 \%, 11 / 1083)$ on stress echocardiography were the most common incidental findings. 

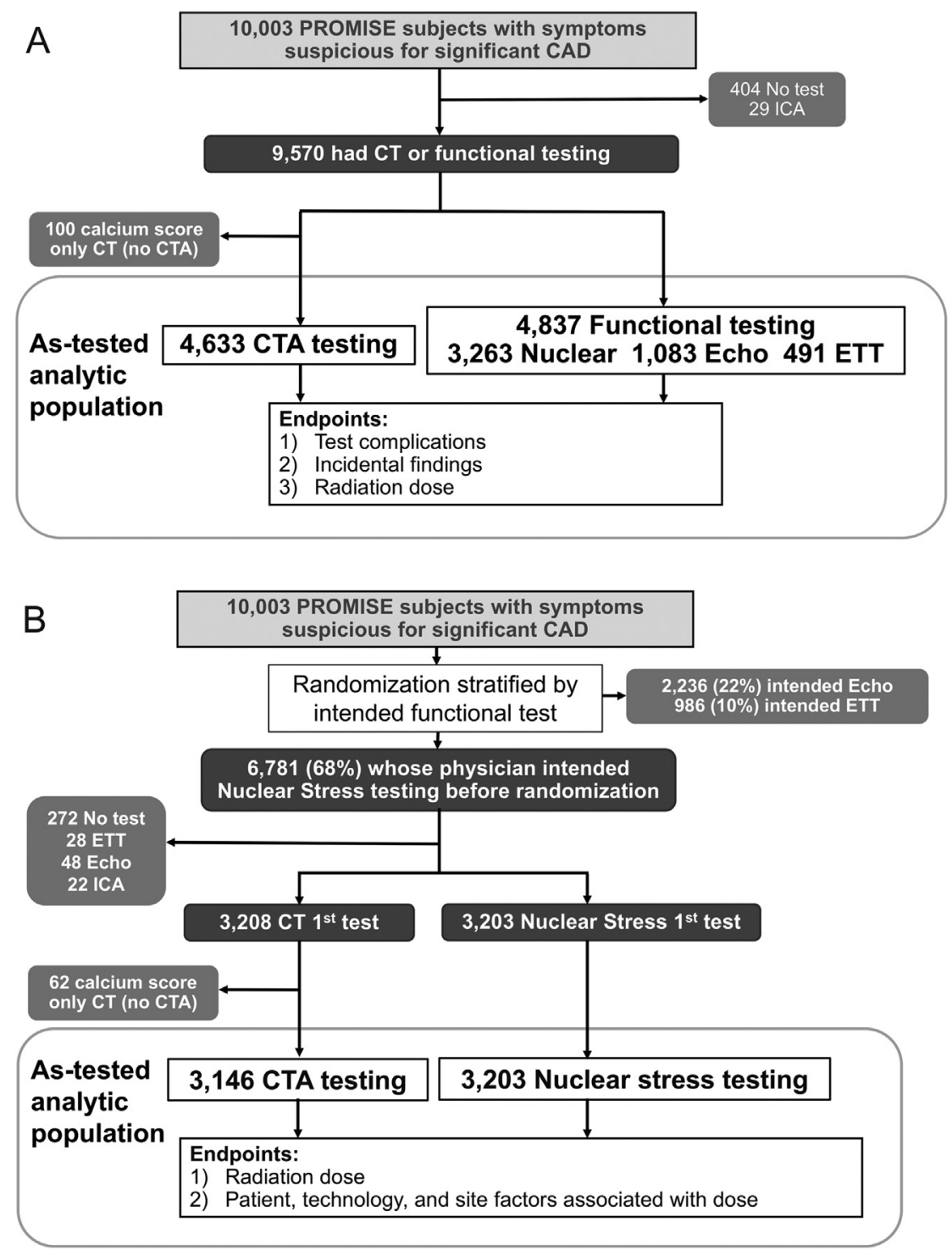

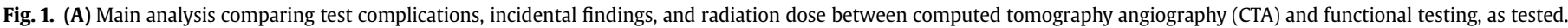

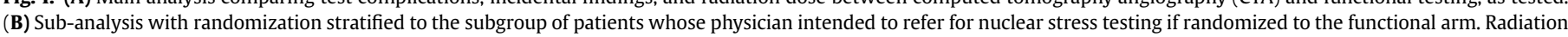

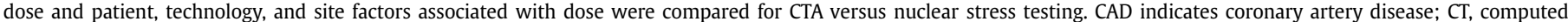
tomography; ETT, exercise tolerance test; ICA, invasive coronary angiography; PROMISE, PROspective Multicenter Imaging Study for Evaluation of Chest Pain.

\subsection{0-day cumulative effective radiation dose - CTA versus functional testing}

As tested, CTA had a lower median ( 10.3 vs. $11.5 \mathrm{mSv}$ ) but higher mean (12.5 vs. $10.6 \mathrm{mSv}$ ) 90-day cumulative effective radiation dose than functional testing (both $\mathrm{p}<0.001$, Table 2 ).

\subsection{Subgroup intended for nuclear stress testing - CTA versus nuclear stress testing}

Of the 10,003 patients enrolled in PROMISE, physicians prespecified nuclear stress testing as the intended test should the patient be randomized to the functional arm in $68 \%(6781 / 10,003$; Fig. 1B). This subgroup was characterized by a significantly higher burden of cardiovascular risk factors and more major adverse cardiovascular events (3.4\% vs. $2.6 \%, \mathrm{p}=0.044$ ) than those prespecified for ETT or stress echocardiography (Supplemental Table 1). Of the 6781 intended for nuclear stress testing, 6.4\% (432) did not complete CTA or nuclear stress testing. The remaining 6349 patients comprised the analytic subgroup intended for nuclear stress testing who were randomized to and completed CTA (3146) or nuclear stress testing (3203). There were similar cardiovascular risk factors and adverse cardiovascular events between those having CTA and nuclear stress testing (Table 1).

\subsection{Index test and 90-day cumulative radiation dose - CTA versus nuclear stress testing}

In the subgroup prespecified for nuclear stress testing, median (IQR) index test effective radiation dose was lower for CTA than for nuclear stress testing, $8.8 \mathrm{mSv}(5.3,14.6)$ versus $12.6 \mathrm{mSv}(11.3$, 14.6), $\mathrm{p}<0.001$ (Fig. 2). Likewise, mean $( \pm \mathrm{SD})$ index test effective dose was also lower for CTA, $10.4 \pm 6.6 \mathrm{mSv}$ versus $14.1 \pm 5.6 \mathrm{mSv}$, $\mathrm{p}<0.001$. A significantly higher percentage of CTA index test doses were $\leq 9 \mathrm{mSv}$ compared to nuclear stress testing (51.2\% vs. $7.0 \%$, $\mathrm{p}<0.001)$. Similar results were observed for 90-day cumulative 
Table 1

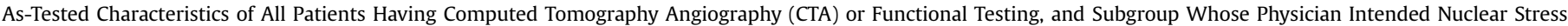
Testing Should the Patient be Randomized to the Functional Testing Arm.

\begin{tabular}{|c|c|c|c|c|}
\hline & \multicolumn{2}{|c|}{ All patients $\mathrm{N}=9470$} & \multicolumn{2}{|c|}{$\begin{array}{l}\text { Subgroup intended for nuclear stress testing } \\
\mathrm{N}=6349\end{array}$} \\
\hline & $\begin{array}{l}\mathrm{CTA} \\
\mathrm{N}=4633\end{array}$ & $\begin{array}{l}\text { Functional testing } \\
\mathrm{N}=4837\end{array}$ & $\begin{array}{l}\text { CTA } \\
\mathrm{N}=3146\end{array}$ & $\begin{array}{l}\text { Nuclear stress testing } \\
\mathrm{N}=3203\end{array}$ \\
\hline Age - mean $\pm S D, y r$ & $60.5 \pm 8.2$ & $61.0 \pm 8.3$ & $61.1 \pm 8.2$ & $61.6 \pm 8.3$ \\
\hline Female sex - no. (\%) & $2392(52 \%)$ & $2587(53 \%)$ & $1694(54 \%)$ & $1786(56 \%)$ \\
\hline Racial/ethnic minority - no. (\%) & $1045(23 \%)$ & $1046(22 \%)$ & $664(21 \%)$ & $626(20 \%)$ \\
\hline \multicolumn{5}{|l|}{ Cardiac Risk factors - no. (\%) } \\
\hline $\mathrm{BMI} \geq 30 \mathrm{~kg} / \mathrm{m}^{2}$ & $2168(47 \%)$ & $2310(48 \%)$ & $1570(50 \%)$ & $1638(51 \%)$ \\
\hline Hypertension & $2991(65 \%)$ & $315(65 \%)$ & $2132(68 \%)$ & $2184(68 \%)$ \\
\hline Diabetes & $970(21 \%)$ & $1061(22 \%)$ & $736(23 \%)$ & $757(24 \%)$ \\
\hline Metabolic syndrome & $1724(37 \%)$ & $1852(38 \%)$ & $1235(39 \%)$ & $1301(41 \%)$ \\
\hline Dyslipidemia & $3121(67 \%)$ & $3282(68 \%)$ & $2111(67 \%)$ & $2183(68 \%)$ \\
\hline Family history premature CAD & $1529(33 \%)$ & $1503(31 \%)$ & $1044(33 \%)$ & $989(31 \%)$ \\
\hline Peripheral arterial disease & $77(1.7 \%)$ & $92(1.9 \%)$ & $57(1.8 \%)$ & $74(2.3 \%)$ \\
\hline CAD equivalent & $1136(25 \%)$ & $1258(26 \%)$ & $862(27 \%)$ & $916(29 \%)$ \\
\hline Current smoker & $822(18 \%)$ & $839(17 \%)$ & $570(18 \%)$ & $573(18 \%)$ \\
\hline History of depression & $909(20 \%)$ & $1044(22 \%)$ & $729(23 \%)$ & $648(21 \%)$ \\
\hline Do not participate in physical activity & $2247(48 \%)$ & 2359 (49\%) & $1654(53 \%)$ & $1672(52 \%)$ \\
\hline Number of risk factors (mean $\pm S D$ ) & $2.4 \pm 1.1$ & $2.4 \pm 1.1$ & $2.4 \pm 1.1$ & $2.4 \pm 1.1$ \\
\hline \multicolumn{5}{|l|}{ Framingham Risk Score } \\
\hline Low risk $(\leq 6 \%)$ & $311(6.7 \%)$ & $346(7.2 \%)$ & 187 (5.9\%) & $211(6.6 \%)$ \\
\hline Intermediate risk (6-20\%) & $2424(52 \%)$ & $2420(50 \%)$ & $1600(51 \%)$ & $1571(49 \%)$ \\
\hline High risk (>20\%) & $1898(41 \%)$ & $2071(43 \%)$ & $1359(43 \%)$ & $1421(44 \%)$ \\
\hline Adverse Cardiovascular Events & $143(3.1 \%)$ & $145(3.0 \%)$ & $108(3.4 \%)$ & $101(3.2 \%)$ \\
\hline
\end{tabular}

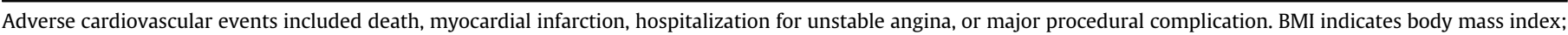
$\mathrm{CAD}$, coronary artery disease.

radiation dose (Fig. 2).

Index test and 90-day cumulative radiation doses remained lower for CTA than nuclear stress testing across all tested subgroups including women, those aged $\geq 65$ years, the obese (BMI $\geq 30 \mathrm{~kg} /$ $\mathrm{m} 2$ ), and those with a resting heart rate $\geq 75$ beats per minute after adjustment for risk factors (all $\mathrm{p}<0.001$, Supplemental Fig. 1 ).

\subsection{Patient factors and CTA radiation dose}

Among the patients who had CTA, men received a higher median index test radiation dose than women (9.3 vs. $8.5 \mathrm{mSv}$, $\mathrm{p}<0.001$ ), the obese had a higher dose than the non-obese (10.5 vs. $7.8 \mathrm{mSv}, \mathrm{p}<0.001$ ), and those with a baseline resting heart rate $\geq 75$ beats per minute had a higher dose than those with a lower heart rate $(9.4$ vs. $8.4 \mathrm{mSv}, \mathrm{p}<0.001)$ (Table 4$)$. Similar significant relationships between 90-day cumulative CTA radiation dose and sex, BMI, and heart rate are enumerated in Supplemental Table 2.

\subsection{Patient factors and nuclear stress testing radiation dose}

Among the patients who had nuclear stress testing, men
(12.7 $\mathrm{mSv}$ vs. $12.4 \mathrm{mSv}$ for women, $\mathrm{p}=0.001$ ) and the obese (12.7 vs. $12.3 \mathrm{mSv}$ for non-obese, $\mathrm{p}<0.001$ ) also had higher median index test radiation dose (Table 4). There was no association between baseline heart rate $\geq 75$ beats per minute and nuclear stress testing dose $(p=0.40)$. For 90 -day cumulative radiation dose, there was not a significant association between age $\geq 65$ years and dose; otherwise, the associations were similar as for index test dose (Supplemental Table 2).

Baseline resting heart rate $\geq 75$ beats per minute had a significantly greater impact on the effective radiation dose of CTA than nuclear stress testing $(\mathrm{p}=0.042)$, with a nonsignificant trend toward a greater impact for obesity and age (Table 4 for index test dose and Supplemental Table 2 for 90-day cumulative dose).

\subsection{Imaging protocol and site experience - CTA versus nuclear stress testing}

Radiation dose varied with site-related factors including the type of CT scanner, annual volume of CTA, and CTA or nuclear stress test protocol (Table 5 ). The $12.2 \%$ of patients who had CTA using an advanced CT scanner had a lower median index test radiation dose

Table 2

Summary of as-tested test complications, potentially significant incidental findings, and 90-day cumulative radiation dose.

\begin{tabular}{|c|c|c|c|}
\hline & \multicolumn{3}{|c|}{ All patients $\mathrm{N}=9470$} \\
\hline & CTA N $=4633$ & Functional testing $\mathrm{N}=4837$ & $\mathrm{p}$ \\
\hline \multicolumn{4}{|l|}{ Complications - no. (\%) } \\
\hline Major & 0 & 0 & \\
\hline Minor & $37(0.8 \%)$ & $27(0.6 \%)$ & 0.15 \\
\hline Incidental findings - no. (\%) & $539(11.6 \%)$ & $34(0.7 \%)$ & $<0.001$ \\
\hline \multicolumn{4}{|l|}{ Radiation dose $-\mathrm{mSv}$} \\
\hline 90-day cumulative & & & $<0.001$ \\
\hline Median (IQR) & $10.3(6.1,17.4)$ & $11.5(0.0,13.8)$ & \\
\hline Mean \pm SD & $12.5 \pm 8.4$ & $10.6 \pm 8.9$ & \\
\hline
\end{tabular}

Major complications included renal failure requiring dialysis, anaphylaxis requiring emergency respiratory or circulatory support, cardiac arrest and death. Minor test complications and incidental findings are enumerated in Table 3. 
Table 3

Index test complications and incidental findings.

\begin{tabular}{|c|c|c|}
\hline Test complications & $\mathrm{N}$ & $\%$ \\
\hline \multicolumn{3}{|l|}{ ETT $(n=491)$} \\
\hline Minor complications & 1 & 0.2 \\
\hline Hypotension & 0 & 0 \\
\hline Drop in systolic BP $>20 \mathrm{mmHg}$ & 0 & 0 \\
\hline Systolic BP $<80 \mathrm{mmHg}$ & 0 & 0 \\
\hline Stress induced symptoms & 0 & 0 \\
\hline Ventricular tachycardia & 0 & 0 \\
\hline Rapid atrial fibrillation & 0 & 0 \\
\hline Hospital admission & 1 & 0.2 \\
\hline Major complications & $\mathbf{0}$ & $\mathbf{0}$ \\
\hline Anaphylaxis & 0 & 0 \\
\hline Renal failure requiring dialysis & 0 & 0 \\
\hline Cardiac arrest & 0 & 0 \\
\hline Death & 0 & 0 \\
\hline \multicolumn{3}{|l|}{ Echo $(n=1083)$} \\
\hline Minor complications & 3 & 0.3 \\
\hline Hypotension & 2 & 0.2 \\
\hline Drop in systolic BP $>20 \mathrm{mmHg}$ & 2 & 0.2 \\
\hline Systolic BP $<80 \mathrm{mmHg}$ & 0 & 0 \\
\hline Stress induced symptoms & 0 & 0 \\
\hline Ventricular tachycardia & 0 & 0 \\
\hline Rapid atrial fibrillation & 0 & 0 \\
\hline Stress induced wall motion abnormality & 0 & 0 \\
\hline Hospital admission & 1 & 0.1 \\
\hline Major complications & $\mathbf{0}$ & $\mathbf{0}$ \\
\hline Anaphylaxis & 0 & 0 \\
\hline Renal failure requiring dialysis & 0 & 0 \\
\hline Cardiac arrest & 0 & 0 \\
\hline Death & 0 & 0 \\
\hline \multicolumn{3}{|l|}{ Nuclear Stress $(n=3263)$} \\
\hline Minor complications & 23 & 0.7 \\
\hline Hypotension & 6 & 0.2 \\
\hline Drop in systolic BP $>20 \mathrm{mmHg}$ & 4 & 0.1 \\
\hline Systolic BP $<80 \mathrm{mmHg}$ & 2 & 0.1 \\
\hline Stress induced symptoms & 4 & 0.1 \\
\hline Ventricular tachycardia & 5 & 0.2 \\
\hline Rapid atrial fibrillation & 0 & 0 \\
\hline Dypridamole/adenosine-related events & 5 & 0.2 \\
\hline Hospital admission & 3 & 0.1 \\
\hline Major complications & $\mathbf{0}$ & $\mathbf{0}$ \\
\hline Anaphylaxis & 0 & 0 \\
\hline Renal failure requiring dialysis & 0 & 0 \\
\hline Cardiac arrest & 0 & 0 \\
\hline Death & 0 & 0 \\
\hline \multicolumn{3}{|l|}{ CTA $(n=4633)$} \\
\hline Minor complications & 37 & 0.8 \\
\hline Mild contrast reaction & 22 & 0.5 \\
\hline Extravasation of contrast & 12 & 0.3 \\
\hline Hemodynamic instability & 3 & 0.1 \\
\hline Acute bronchospasm & 0 & 0 \\
\hline Hospital admission & 0 & 0 \\
\hline Major complications & $\mathbf{0}$ & $\mathbf{0}$ \\
\hline Anaphylaxis & 0 & 0 \\
\hline Renal failure requiring dialysis & 0 & 0 \\
\hline Cardiac arrest & 0 & 0 \\
\hline Death & 0 & 0 \\
\hline Incidental findings & $\mathrm{N}$ & $\%$ \\
\hline Echo $(n=1083)$ & 21 & 1.9 \\
\hline Moderate or large pericardial effusion or tamponade & 0 & 0 \\
\hline Moderate or severe aortic stenosis & 2 & 0.2 \\
\hline Moderate or severe mitral stenosis & 0 & 0 \\
\hline Hypertrophic obstructive cardiomyopathy & 2 & 0.2 \\
\hline Endocarditis & 0 & 0 \\
\hline Mitral prolapse & 6 & 0.6 \\
\hline Aortic root aneurysm & 3 & 0.3 \\
\hline Moderate or severe aortic insufficiency & 3 & 0.3 \\
\hline Moderate or severe mitral regurgitation & 11 & 1.0 \\
\hline Moderate or severe pulmonary hypertension & 1 & 0.1 \\
\hline Nuclear Stress $(n=3263)$ & 13 & 0.4 \\
\hline Breast uptake & 11 & 0.3 \\
\hline Lung uptake & 1 & 0.03 \\
\hline Axillary uptake & 1 & 0.03 \\
\hline
\end{tabular}

Table 3 (continued)

\begin{tabular}{lll}
\hline Incidental findings & $\mathrm{N}$ & $\%$ \\
\hline CTA $(\mathbf{n}=\mathbf{4 6 3 3})$ & $\mathbf{5 3 9}$ & $\mathbf{1 1 . 6}$ \\
Coronary anomaly & 71 & 1.5 \\
Lung nodule & 437 & 9.4 \\
$\quad$ Size of largest & & \\
$\quad \leq 4 \mathrm{~mm}$ & 201 & 4.3 \\
$5-7 \mathrm{~mm}$ & 161 & 3.5 \\
$\quad \geq 8 \mathrm{~mm}$ & 74 & 1.6 \\
Pulmonary embolism & 4 & 0.1 \\
Pneumonia & 9 & 0.2 \\
Aortic aneurysm & 89 & 1.9 \\
Aortic dissection & 8 & 0.2 \\
Hiatal hernia & 358 & 7.7 \\
\hline
\end{tabular}

BP indicates blood pressure; CTA, computed tomography angiography; ETT, exercise tolerance test.

than those who had CTA on a standard CT scanner (5.5 vs. $9.3 \mathrm{mSv}$, $\mathrm{p}<0.001$ ). Most CTA (74.3\%) was performed using a prospectively ECG-triggered scan, which had a lower index radiation dose than retrospectively ECG-gated scans (6.6 vs. $9.8 \mathrm{mSv}, \mathrm{p}<0.001$ ). Most nuclear stress testing was performed with a 1-day Tc $99 \mathrm{~m}$ Sestamibi or Tetrofosmin rest/stress (77\%, 2462/3203; index test, $12.2 \mathrm{mSv}$ ) or stress/rest (3.2\%, 103/3203; index test, $12.7 \mathrm{mSv})$ protocol, with a lower index test radiation dose $(\mathrm{p}<0.001)$ than 2 day protocols and 1-day protocols including a Thallium 201 tracer (Table 5). Median CTA index test dose was inversely associated with the site's self-reported annual CTA volume (12.3 mSv for sites performing 1-100 CTAs/year, $10.4 \mathrm{mSv}$ for 101-500 CTAs/year, and $6.9 \mathrm{mSv}$ for $>500$ CTAs/year, $\mathrm{p}$ for trend $<0.001$ ). Similar relationships were seen for 90 -day cumulative radiation dose. There was no linear association between site annual volume of nuclear stress testing and radiation dose.

\section{Discussion}

The PROMISE trial found that, in stable outpatients with suspected CAD, anatomic CTA and functional testing strategies resulted in a similar rate of cardiovascular events. ${ }^{2}$ Given this state of equipoise, test safety should help guide test choice. In the current prespecified as-tested randomized comparison of test safety in the PROMISE trial, CTA and functional testing had a similar very low $<1 \%$ rate of minor test complications and no major complications. CTA detected substantially more potentially significant incidental findings. CTA had similar 90-day cumulative dose to functional testing overall. However, in the subgroup of patients whom physicians intended to refer for nuclear stress testing if randomly assigned to the functional testing arm, CTA had significantly lower index test and 90-day cumulative radiation doses than nuclear stress testing, independent of patient characteristics including obesity and heart rate. For both CTA and nuclear stress testing, radiation dose varied substantially with equipment, imaging protocol, and patient characteristics.

This randomized comparison of test safety between CTA and functional testing extends previous retrospective cohorts demonstrating similar low complication rates for each test. For CTA, contrast reactions $(0.4 \%)$ and local extravasation (0.3\%) were the most common minor complications and occurred at similar rates as reported elsewhere. ${ }^{17-20}$ Likewise, functional testing complications were similarly rare $(0.6 \%)$ and within the range previously reported. ${ }^{21}$ These data suggest that CTA and functional testing are similarly safe with only rare, and usually minor, test complications.

Our findings confirm previous reports that incidental findings are substantially more frequent on CTA as compared to functional testing. ${ }^{3,22-24}$ Findings with the potential to cause chest pain such as coronary anomalies, pneumonia, pulmonary embolism, and 


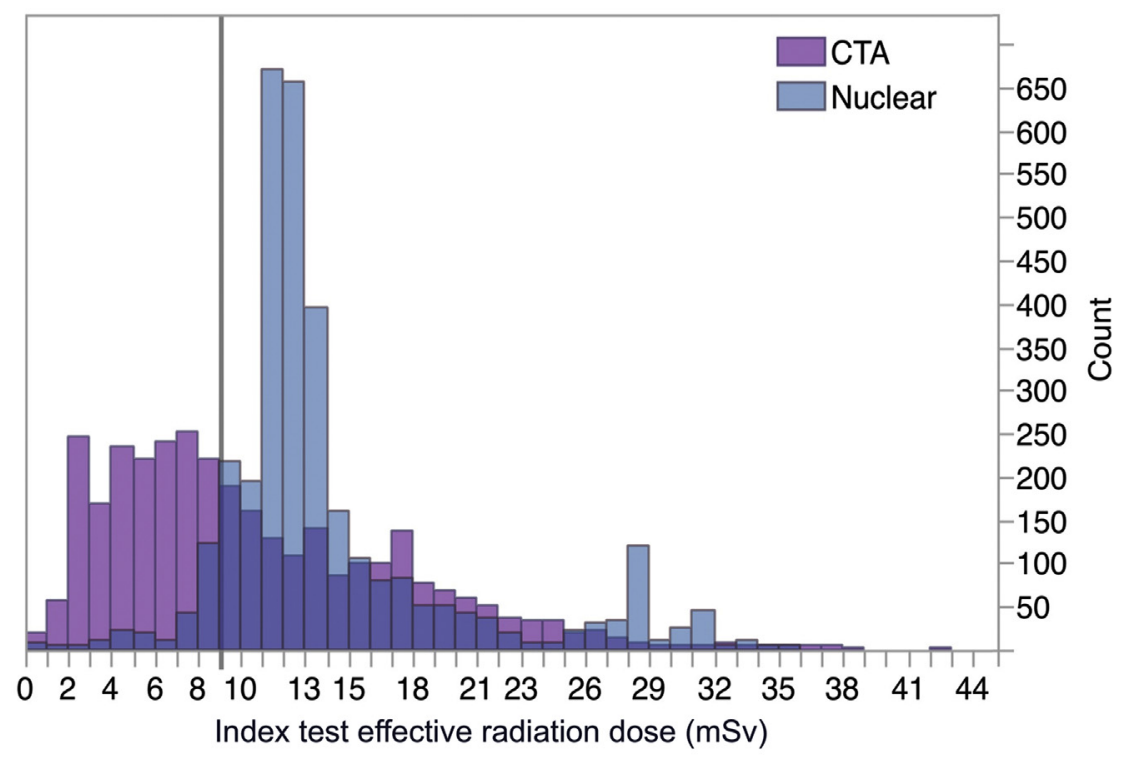

\begin{tabular}{|c|c|c|c|}
\hline & $\begin{array}{r}\text { Subgroup intended for nuclear } \\
\text { stress testing } \\
N=6349\end{array}$ & \\
\hline & $\begin{array}{c}\text { CTA } \\
\mathrm{N}=3146\end{array}$ & $\begin{array}{c}\text { Nuclear } \\
\mathrm{N}=3203\end{array}$ & $\mathrm{p}$ \\
\hline Index test $-\mathrm{mSv}$ & & & $<0.001$ \\
\hline Median (IQR) & $8.8(5.3,14.6)$ & $12.6(11.3,14.6)$ & \\
\hline Mean \pm SD & $10.4 \pm 6.6$ & $14.1 \pm 5.6$ & \\
\hline$\leq 9$ mSv, no. (\%) & $1611(51.2 \%)$ & $223(7.0 \%)$ & $<0.001$ \\
\hline 90-day cumulative - mSv & & & $<0.001$ \\
\hline Median (IQR) & $11.6(6.7,18.5)$ & $13.1(11.5,19.4)$ & \\
\hline Mean \pm SD & $13.6 \pm 9.2$ & $16.2 \pm 7.7$ & \\
\hline
\end{tabular}

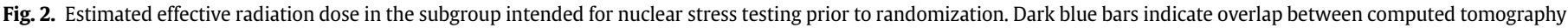

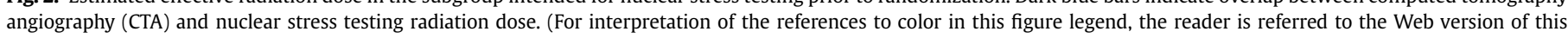
article.)

aortic dissection were found in $2 \%$ of patients having CTA. Put in context, only $12 \%$ of PROMISE patients had obstructive CAD on CTA. ${ }^{25}$ Nevertheless most incidental findings were truly incidental and would not be expected to cause chest pain, most commonly lung nodules seen in 9\%. In PROMISE the outcomes of incidental findings and noncardiac downstream testing was not collected, which precludes an analysis of their impact. However, other studies have suggested that follow-up of lung nodules incidentally detected on CTA can prevent lung cancer mortality, but at high cost. ${ }^{6,24,26}$
Goehler et al. found that follow-up of lung nodules incidentally detected on coronary CTA by the 2005 Fleischner Society guidelines resulted in a $4.6 \%$ relative reduction in lung cancer mortality, but at an estimated cost per quality adjusted life year (QALY) of $\$ 154,700$. $^{6,27}$ On the other hand, for high risk heavy smokers the National Lung Screening Trial found that screening chest CT and follow-up of lung nodules was cost-effective at $\$ 81,000$ per QALY. $^{28}$ Clinical trials are necessary to determine in which nodule and patient risk categories follow-up is necessary. The Watch The Spot

Table 4

Index test radiation effective dose by patient characteristics in subgroup intended for nuclear stress testing.

\begin{tabular}{|c|c|c|c|c|c|c|c|c|c|}
\hline \multirow[t]{2}{*}{ Patient characteristic } & \multicolumn{4}{|c|}{ Nuclear } & \multicolumn{4}{|l|}{ CTA } & \multirow[t]{2}{*}{$\mathrm{p}$ for effect on nuclear vs CTA $\mathrm{R}^{2}$} \\
\hline & $\mathrm{N}$ & Median (IQR) in mSv & Mean \pm SD in $\mathrm{mSv}$ & $\mathrm{p}$ & $\mathrm{N}$ & Median (IQR) in mSv & Mean \pm SD in $\mathrm{mSv} p$ & $\mathrm{p}$ & \\
\hline Age & & & & 0.001 & & & & 0.087 & 0.072 \\
\hline$<65$ years & 2159 & $12.7(11.3,15.0)$ & $14.3 \pm 5.7$ & & 2178 & $9.1(5.4,14.7)$ & $10.6 \pm 6.6$ & & \\
\hline$\geq 65$ years & 1044 & $12.3(11.2,13.8)$ & $13.5 \pm 5.2$ & & 968 & $8.4(5.1,14.5)$ & $10.3 \pm 6.7$ & & \\
\hline Sex & & & & $<0.001$ & & & & $<0.001$ & 0.82 \\
\hline Male & 1417 & $12.7(11.4,15.2)$ & $14.4 \pm 5.7$ & & 1452 & $9.3(5.5,15.9)$ & $11.1 \pm 7.0$ & & \\
\hline Female & 1786 & $12.4(11.2,14.1)$ & $13.8 \pm 5.4$ & & 1694 & $8.5(5.2,13.5)$ & $9.9 \pm 6.2$ & & \\
\hline BMI & & & & $<0.001$ & & & & $<0.001$ & 0.10 \\
\hline Not Obese $(<30 \mathrm{~kg} / \mathrm{m} 2)$ & 1537 & $12.3(11.2,13.7)$ & $13.8 \pm 5.7$ & & 1556 & $7.8(4.5,12.6)$ & $9.2 \pm 6.11$ & & \\
\hline Obese $(\geq 30 \mathrm{~kg} / \mathrm{m} 2)$ & 1638 & $12.7(11.4,15.7)$ & $14.3 \pm 5.4$ & & 1570 & $10.5(6.5,6.1)$ & $11.8 \pm 6.9$ & & \\
\hline Heart rate $^{a}$ & & & & 0.40 & & & & $<0.001$ & 0.042 \\
\hline$<75$ bpm & 1895 & $12.6(11.3,14.6)$ & $14.1 \pm 5.6$ & & 1859 & $8.4(5.0,13.8)$ & $10.1 \pm 6.7$ & & \\
\hline$\geq 75 \mathrm{bpm}$ & 1300 & $12.5(11.3,14.5)$ & $14.0 \pm 5.4$ & & 1279 & $9.4(5.8,15.6)$ & $11.0 \pm 6.5$ & & \\
\hline
\end{tabular}

BMI indicates body mass index; CTA, computed tomography angiography.

a Resting heart rate was recorded at the enrollment physical examination. 
Table 5

Radiation effective dose by technology, protocol, and site factors in subgroup intended for nuclear stress testing.

\begin{tabular}{|c|c|c|c|c|c|c|c|}
\hline \multirow[t]{2}{*}{ Protocol or site characteristic } & \multirow[t]{2}{*}{$\mathrm{N}$} & \multicolumn{3}{|c|}{ Index test radiation dose } & \multicolumn{3}{|c|}{ 90-day cumulative radiation dose } \\
\hline & & $\begin{array}{l}\text { Median (IQR) in } \\
\mathrm{mSv}\end{array}$ & $\begin{array}{l}\text { Mean } \pm \mathrm{SD} \text { in } \\
\mathrm{mSv}\end{array}$ & $\mathrm{p}$ & $\begin{array}{l}\text { Median (IQR) in } \\
\mathrm{mSv}\end{array}$ & $\begin{array}{l}\text { Mean } \pm S D \text { in } \\
\mathrm{mSv}\end{array}$ & $\mathrm{p}$ \\
\hline All CTA & 3146 & $8.8(5.3,14.6)$ & $10.4 \pm 6.6$ & & $11.6(6.7,18.5)$ & $13.6 \pm 9.2$ & \\
\hline CT scanner & & & & $<0.001$ & & & $<0.001$ \\
\hline Standard & 2725 & $9.3(5.9,15.4)$ & $11.0 \pm 6.7$ & & $11.0(6.7,17.6)$ & $12.9 \pm 8.4$ & \\
\hline Advanced & 379 & $5.5(4.0,8.8)$ & $7.0 \pm 4.9$ & & $6.1(4.2,11.6)$ & $8.6 \pm 6.7$ & \\
\hline CTA scan mode & & & & $<0.001$ & & & $<0.001$ \\
\hline Retrospective & 797 & $9.8(6.2,15.9)$ & $11.2 \pm 6.7$ & & $11.5(6.9,17.9)$ & $13.2 \pm 8.5$ & \\
\hline Prospective & 2307 & $6.6(4.5,10.3)$ & $8.3 \pm 6.0$ & & $7.7(4.8,13.2)$ & $9.9 \pm 7.5$ & \\
\hline Annual CTA site volume & & & & $<0.001$ & & & 0.0004 \\
\hline$<25$ th percentile $(1-100)$ & 337 & $12.3(7.9,17.7)$ & $12.8 \pm 6.4$ & & $13.2(7.9,18.9)$ & $14.5 \pm 8.7$ & \\
\hline $25^{\text {th }}-75$ th percentile $(100-500)$ & 1077 & $10.4(5.4,17.4)$ & $11.8 \pm 7.5$ & & $12.0(6.9,19.1)$ & $13.5 \pm 8.9$ & \\
\hline$>75$ th percentile $(>500)$ & 1023 & $6.9(4.1,11.8)$ & $8.7 \pm 6.2$ & & $8.0(4.5,14.5)$ & $10.7 \pm 8.3$ & \\
\hline All nuclear stress testing & 3203 & $12.6(11.3,14.6)$ & $14.1 \pm 5.6$ & & $13.1(11.5,19.4)$ & $16.2 \pm 7.7$ & \\
\hline Nuclear stress protocol & & & & $<0.001$ & & & $<0.0001$ \\
\hline $\begin{array}{l}\text { 1. 2-day rest stress dual isotope (Thallium } 201 \text { rest/Tc99m Sestamibi stress or } \\
\text { Tetrofosmin stress) }\end{array}$ & 302 & $28.2(27.4,30.4)$ & $28.3 \pm 3.0$ & & $28.3(27.7,31.5)$ & $29.8 \pm 4.9$ & \\
\hline 2. 1-day rest/stress Tc99m Sestamibi or Tetrofosmin & 2462 & $12.2(11.2,13.3)$ & $12.3 \pm 2.3$ & & $12.5(11.3,14.0)$ & $13.4 \pm 4.4$ & \\
\hline 3. 2-day rest/stress Tc99m Sestamibi or Tetrofosmin & 132 & $19.7(18.2,21.3)$ & $19.7 \pm 3.8$ & & $20.4(18.3,25.0)$ & $21.8 \pm 6.3$ & \\
\hline 4. 1-day stress/rest Tc99m Sestamibi or Tetrofosmin & 103 & $12.7(12.7,12.7)$ & $12.5 \pm 1.4$ & & $12.7(12.7,13.8)$ & $13.9 \pm 3.7$ & \\
\hline 5. 1-day stress-redistribution Thallium 201 optional $18-24$ h images & 32 & $21.2(20.7,21.4)$ & $21.5 \pm 2.0$ & & $21.2(20.7,21.4)$ & $22.0 \pm 3.2$ & \\
\hline Annual nuclear site volume & & & & $<0.001$ & & & 0.051 \\
\hline$<25$ th percentile $(<795)$ & 550 & $13.0(10.9,22.2)$ & $16.1 \pm 7.4$ & & $13.1(11.1,27.1)$ & $17.6 \pm 8.9$ & \\
\hline $25^{\text {th }}-75$ th percentile $(795-3393)$ & 1277 & $12.1(11.3,13.3)$ & $13.3 \pm 4.3$ & & $12.5(11.4,14.4)$ & $14.3 \pm 5.8$ & \\
\hline >75th percentile (>3393) & 1014 & $12.8(11.0,15.3)$ & $14.2 \pm 5.9$ & & $13.2(11.4,17.3)$ & $15.3 \pm 6.9$ & \\
\hline
\end{tabular}

P-values are for comparisons of medians between each category of imaging protocol or site factor.

Trial, an ongoing 40,000-subject pragmatic comparative effectiveness trial, aims to assess whether a less intensive follow-up strategy is safe and cost-effective. ${ }^{29}$ In 2017 the Fleischner Society released updated guidelines which raise the size threshold for lung nodule follow-up and should result in substantially less downstream testing. ${ }^{30}$

PROMISE previously reported that patients randomized to CTA had a lower median (10.3 vs. $11.2 \mathrm{mSv}$ ) but higher mean (12.0 vs. $10.1 \mathrm{mSv}$ ) 90-day cumulative effective radiation dose than patients randomized to functional testing, but this included those who were not tested. ${ }^{7}$ As tested in the current analysis, there was essentially the same relationship with CTA having lower median (10.3 vs. $11.5 \mathrm{mSv}$ ) but higher mean ( $12.5 \mathrm{vs} .10 .6 \mathrm{mSv}$ ) 90-day cumulative effective radiation dose than functional testing. This is a difficult comparison because one-third of functional tests (i.e., ETT or stress echocardiography) delivered no radiation dose. It is important to note that, if these functional tests with no radiation dose are chosen over nuclear stress testing, the exam requiring radiation will obviously have substantially greater dose.

The choice to pursue nuclear stress testing over other functional tests for a given patient is multifactorial, incorporating cardiovascular risk profile, exercise capacity, ECG, cost, and local availability in addition to radiation dose. Despite the fact that nuclear stress testing necessarily gives more radiation dose, physicians chose nuclear stress testing over ETT or stress echocardiography in twothirds of PROMISE patients. This reflects contemporary practice in the United States, where nuclear stress testing is the most common functional imaging test. ${ }^{31,32}$ Furthermore, PROMISE patients intended for nuclear stress testing were a high risk group with greater cardiovascular risk factors and major adverse cardiovascular events than patients intended for other functional tests. For these reasons, we focused our second analysis of radiation dose on the randomization strata of patients whom their physicians intended to refer for nuclear stress testing if randomized to the functional arm. In this group, CTA's index test effective radiation dose was $30 \%$ lower than nuclear stress testing (median 8.8 vs. $12.6 \mathrm{mSv}, \mathrm{p}<0.001$ ). CTA's lower radiation dose than nuclear stress testing was durable across all tested subgroups, including patients traditionally difficult to image with CTA, such as the obese and those with a resting heart rate $\geq 75$ beats per minute.

\subsection{Opportunities to lower radiation dose}

PROMISE was designed to reflect actual care of patients with stable chest pain in a broad cross-section of 193 North American sites during the enrollment period from 2010 through 2013. The radiation dose data should be interpreted in the context of ongoing technological advances for both CTA and nuclear stress testing. The CTA index test radiation dose in PROMISE (median $8.8 \mathrm{mSv}$ ) was lower than the median $12 \mathrm{mSv}$ reported in the 2007 PROTECTION I (Prospective Multicenter Study on Radiation Dose Estimates of Cardiac CT Angiography in Daily Practice I) survey of 21 university and 29 community international CTA sites, ${ }^{33}$ reflecting advances in CT dose reduction technology and increasing awareness of the risks of ionizing radiation. ${ }^{34}$ The majority (88\%) of PROMISE CTA was performed on standard 64-slice CT scanners now ubiquitous in community practice. However, $12 \%$ were performed on newer advanced CT scanners, and in this group the index test dose was substantially lower (median $5.5 \mathrm{mSv}$ ). Sites with the most experience, as measured by annual CTA volume, also had a low dose (median $6.9 \mathrm{mSv}$ ). In the contemporaneous European SCOT-HEART (Scottish Computed Tomography of the Heart) trial, CTA performed at 4 expert sites with advanced 320 -slice CT scanners found a slightly lower median dose of $4.1 \mathrm{mSv}^{3}$ Since the completion of PROMISE and SCOT-HEART, CT manufacturers have released new CT scanners and techniques that promise to further reduce dose, with expert sites reporting CTA doses $<3 \mathrm{mSv}^{34-36}$

Opportunities also exist to improve on our observed radiation dose with nuclear testing. For nuclear stress testing, the median index test dose was $12.6 \mathrm{mSv}$, similar to the dose reported in contemporaneous surveys of US nuclear laboratories, with $14.9 \mathrm{mSv}$ reported in 2012-2013 by the Intersocietal Accreditation Commission $^{37}$ and $11.6 \mathrm{mSv}$ in the 2013 International Atomic Energy Agency Nuclear Cardiology Protocols Study (INCAPS). ${ }^{38}$ These 
doses fail to reach the American Society of Nuclear Cardiology's goal of at least $50 \%$ of nuclear stress tests having a dose $\leq 9 \mathrm{mSv} .{ }^{16}$ In PROMISE, only $7 \%$ of nuclear stress studies were $\leq 9 \mathrm{mSv}$, in comparison to $51 \%$ of CTA. While nuclear stress testing is substantially more mature than CTA, there is also room for dose reduction. Radiation dose is approximately $20 \%$ higher in US laboratories compared to non-US laboratories, and this gap could in part be bridged by following best practices such as avoiding dual-isotope protocols, using weight-adjusted doses, performing stress-only imaging in appropriate patients, and using advanced singlephoton emission computed tomography (SPECT) cameras. ${ }^{16,38}$ Three expert sites not in PROMISE injecting a low dose of ${ }^{99 \mathrm{~m}} \mathrm{Tc}$ and using stress-only imaging with advanced SPECT cameras reported doses as low as $1.2 \mathrm{mSv}$ in 101 patients, although obese patients (who made up half of the PROMISE population) were excluded ${ }^{39}$ In PROMISE, most nuclear stress testing was performed with a SPECT ${ }^{99 \mathrm{~m}} \mathrm{Tc}$ tracer-based protocol, with only $10 \%$ having a SPECT study including a ${ }^{201}$ Th tracer. Positron emission tomography (PET) stress testing, which was not used in PROMISE, may provide lower dose examinations, with expert PET sites reporting low radiation doses on the order of $3 \mathrm{mSv}$ for ${ }^{82} \mathrm{Rb},{ }^{13} \mathrm{~N}$-ammonia, and ${ }^{15} \mathrm{O}$-water based protocols. ${ }^{40}$ Stress perfusion cardiovascular magnetic resonance was not performed in PROMISE, but could have provided an alternative with no radiation dose. ${ }^{41}$

CTA had a 3.8-mSv lower median effective dose than nuclear stress testing. This dose difference is roughly equivalent to 382 view chest radiographs and on the order of the approximately 3$\mathrm{mSv}$ average annual dose from natural background radiation in the United States. ${ }^{4}$ This difference is low and unlikely to be of clinical significance for individual patients. ${ }^{42}$ Nevertheless, the most conservative approach is to treat any radiation dose as increasing the risk of cancer on a population basis. ${ }^{43,44}$ In the United States, nuclear stress testing accounts for $22 \%$ of the total population effective dose from all diagnostic imaging tests including non-cardiac imaging, and so choosing CTA as a lower dose alternative for some patients could have a substantial impact. ${ }^{45}$ Alternative tests with no radiation dose (i.e., ETT or stress echocardiography) should also be considered in appropriate patients.

\subsection{Limitations}

We quantified radiation exposure using the effective dose, a commonly used metric in the radiation dose literature. ${ }^{4,12}$ Estimates of the effective dose of CTA were based on the European Commission and American Association of Physicists in Medicine chest $\mathrm{k}$-factor of $0.014 \mathrm{mSv} \mathrm{mGy}^{-1} \mathrm{~cm}^{-1}$, the most commonly used conversion factor for cardiac CT. ${ }^{13,46}$ Alternative k-factors have been proposed and would result in different dose estimates. ${ }^{47}$ Effective dose calculations make generic assumptions of patient size, gender, and age, specifically by averaging dose between male and female non-obese phantoms across a population of all ages. ${ }^{48,49}$ As such, effective dose is a poor indicator of an individual patient's risk and is better suited to comparisons between populations having different tests as in our study. Due to the averaging intrinsic to its definition, comparisons of effective dose across categories of gender, BMI, and age, while commonly reported, go beyond the scope of its intended use. We excluded 100 subjects who had a calcium score-only CT (no CTA). Calcium score CT has substantially lower radiation dose than CTA, and including these subjects would have resulted in a lower index CTA dose estimate. Information about non-cardiovascular testing and outcomes such as cancer were not collected. As a result, the ultimate clinical significance of incidental findings and the effectiveness of identifying them are beyond the scope of the data.

\subsection{Conclusions}

Both CTA and functional stress testing are safe, with minor complications in $<1 \%$. CTA detects more potentially significant incidental findings. CTA's lower radiation dose than nuclear stress testing, independent of patient characteristics, makes it an attractive alternative test for the evaluation of stable chest pain. Radiation dose varies substantially with equipment, site experience, and imaging protocol, indicating opportunities to further reduce dose.

\section{Funding and disclosures}

This work was supported by National Heart, Lung, and Blood Institute grants R01HL098237, R01HL098236, R01HL098305, R01HL098235, and R01HL109711. Dr. Lu's effort was supported by the American Roentgen Ray Society Scholarship. Dr. Ghoshhajra has received research grants from Siemens Healthcare. Dr. Einstein has received research grants to Columbia University from GE Healthcare, Philips Healthcare, and Toshiba America Medical Systems. Dr. Mark has received grant support from Eli Lilly, Bristol-Myers Squibb, Gilead Sciences, AGA Medical, Merck, Oxygen Biotherapeutics, and AstraZeneca as well as personal fees from Medtronic, CardioDx, and St. Jude Medical. Dr. Douglas has received grants from HeartFlow and GE Healthcare. Dr. Hoffmann has received grants from HeartFlow and Siemens Healthcare, and is a consultant to HeartFlow. All other authors have reported that they have no relationships relevant to the contents of this paper to disclose. The funding sources had no role in the design of the study, data analysis, or drafting of the manuscript. The content of this manuscript is solely the responsibility of the authors and does not necessarily reflect the views of the National Heart, Lung, and Blood Institute.

\section{Appendix A. Supplementary data}

Supplementary data related to this article can be found at http:// dx.doi.org/10.1016/j.jcct.2017.08.005.

\section{References}

1. Ladapo JA, Blecker S, Douglas PS. Physician decision making and trends in the use of cardiac stress testing in the United States: an analysis of repeated crosssectional data. Ann Intern Med. 2014;161:482-490.

2. Douglas PS, Hoffmann U, Patel MR, et al. Outcomes of anatomical versus functional testing for coronary artery disease. N Engl J Med. 2015;372: $1291-1300$.

3. Investigators SCOT-HEART. CT coronary angiography in patients with suspected angina due to coronary heart disease (SCOT-HEART): an open-label, parallel-group, multicentre trial. Lancet. 2015;385:2383-2391.

4. Mettler Jr FA, Huda W, Yoshizumi TT, Mahesh M. Effective doses in radiology and diagnostic nuclear medicine: a catalog. Radiology. 2008;248:254-263.

5. Lehman SJ, Abbara S, Cury RC, et al. Significance of cardiac computed tomography incidental findings in acute chest pain. Am J Med. 2009;122:543-549.

6. Goehler A, McMahon PM, Lumish HS, et al. Cost-effectiveness of follow-up of pulmonary nodules incidentally detected on cardiac computed tomographic angiography in patients with suspected coronary artery disease. Circulation. 2014;130:668-675.

7. Douglas PS, Hoffmann U, Lee KL, et al. PROMISE Investigators. PROspective Multicenter Imaging Study for Evaluation of chest pain: rationale and design of the PROMISE trial. Am Heart J. 2014;167:796-803. e1.

8. Abbara S, Arbab-Zadeh A, Callister TQ et al. SCCT guidelines for performance of coronary computed tomographic angiography: a report of the Society of Cardiovascular Computed Tomography Guidelines Committee. J Cardiovasc Comput Tomogr. 2009;3:190-204.

9. Raff GL, Abidov A, Achenbach S, et al. Society of Cardiovascular Computed Tomography. SCCT guidelines for the interpretation and reporting of coronary computed tomographic angiography. J Cardiovasc Comput Tomogr. 2009;3: $122-136$.

10. Chen J, Einstein AJ, Fazel R, et al. Cumulative exposure to ionizing radiation from diagnostic and therapeutic cardiac imaging procedures: a populationbased analysis. J Am Coll Cardiol. 2010;56:702-711.

11. Gerber TC, Carr JJ, Arai AE, et al. Ionizing radiation in cardiac imaging: a science 
advisory from the American heart association committee on cardiac imaging of the council on clinical cardiology and committee on cardiovascular imaging and intervention of the council on cardiovascular radiology and intervention. Circulation. 2009;119:1056-1065.

12. Einstein AJ, Moser KW, Thompson RC, Cerqueira MD, Henzlova MJ. Radiation dose to patients from cardiac diagnostic imaging. Circulation. 2007;116: 1290-1305.

13. American Association of Physicists in Medicine. The Measurement, Reporting and Management of Radiation Dose in CT: Report of AAPM Task Group 23 of the Diagnostic Imaging Council CT Committee. 2008. Available at:http://www.aapm. org/pubs/reports/rpt_96.pdf.

14. Mattsson S, Johansson L, Leide Svegborn S, et al, on behalf of ICRP. Radiation dose to patients from radiopharmaceuticals: a compendium of current information related to frequently used substances: ICRP Publication 128. Ann ICRP. 2015;44(2 Suppl):7-321.

15. Cousins C, Miller DL, Bernardi G, et al. International commission on radiological protection. Radiological protection in cardiology: ICRP publication 120. Ann ICRP. 2013;42:1-125.

16. Cerqueira MD, Allman KC, Ficaro EP, et al. Recommendations for reducing radiation exposure in myocardial perfusion imaging. J Nucl Cardiol. 2010;17: 709-718.

17. Dean KE, Starikov A, Giambrone A, Hentel K, Min R, Loftus M. Adverse reactions to intravenous contrast media: an unexpected discrepancy between inpatient and outpatient cohorts. Clin Imaging. 2015;39:863-865.

18. Wang CL, Cohan RH, Ellis JH, Adusumilli S, Dunnick NR. Frequency, management, and outcome of extravasation of nonionic iodinated contrast medium in 69,657 intravenous injections. Radiology. 2007:243:80-87.

19. Cohan RH, Bullard MA, Ellis JH, et al. Local reactions after injection of iodinated contrast material: detection, management, and outcome. Acad Radiol. 1997;4: 711-718.

20. Federle MP, Chang PJ, Confer S, Ozgun B. Frequency and effects of extravasation of ionic and nonionic CT contrast media during rapid bolus injection. Radiology. 1998;206:637-640.

21. Dilsizian V, Gewirtz H, Paivanas N, et al. Serious and potentially life threatening complications of cardiac stress testing: physiological mechanisms and management strategies. J Nucl Cardiol. 2015;22:1198-1213.

22. Gedik GK, Ergun EL, Aslan M, Caner B. Unusual extracardiac findings detected on myocardial perfusion single photon emission computed tomography studies with Tc-99m sestamibi. Clin Nucl Med. 2007;32:920-926.

23. Alkhouli M, Sandhu P, Wiegers SE, Patil P, Panidis J, Pursnani A. Extracardiac findings on routine echocardiographic examinations. J Am Soc Echocardiogr. 2014;27:540-546.

24. Onuma Y, Tanabe K, Nakazawa G, et al. Noncardiac findings in cardiac imaging with multidetector computed tomography. J Am Coll Cardiol. 2006;48: 402-406.

25. Pagidipati NJ, Hemal K, Coles A, et al. Sex differences in functional and ct angiography testing in patients with suspected coronary artery disease. J Am Coll Cardiol. 2016:67:2607-2616.

26. Machaalany J, Yam Y, Ruddy TD, et al. Potential clinical and economic consequences of noncardiac incidental findings on cardiac computed tomography. I Am Coll Cardiol. 2009;54:1533-1541.

27. MacMahon H, Austin JH, Gamsu G, et al. Guidelines for management of small pulmonary nodules detected on CT scans: a statement from the Fleischner Society. Radiology. 2005;237:395-400.

28. Black WC, Gareen IF, Soneji SS, et al. National lung screening trial research team. Cost-effectiveness of CT screening in the national lung screening trial. N Engl J Med. 2014;371:1793-1802.

29. The Watch the Spot Trial (WTS). 2016. Available at: https://clinicaltrials.gov/ ct2/show/NCT02623712.

30. MacMahon H, Naidich DP, Goo JM, et al. Guidelines for management of incidental pulmonary nodules detected on CT images: from the Fleischner Society
2017. Radiology. 2017 Feb 23:161659. http://dx.doi.org/10.1148/ radiol.2017161659 [Epub ahead of print]

31. Levin DC, Parker L, Halpern EJ, Rao VM. Recent trends in imaging for suspected coronary artery disease: what is the best approach? J Am Coll Radiol. 2016;13: $381-386$.

32. Shreibati JB, Baker LC, Hlatky MA. Association of coronary CT angiography or stress testing with subsequent utilization and spending among Medicare beneficiaries. JAMA. 2011;306:2128-2136.

33. Hausleiter J, Meyer T, Hermann F, et al. Estimated radiation dose associated with cardiac CT angiography. JAMA. 2009;301:500-507.

34. Ghoshhajra BB, Engel LC, Major GP, et al. Evolution of coronary computed tomography radiation dose reduction at a tertiary referral center. Am J Med. 2012:125:764-772.

35. Bom MJ, van der Zee PM, Cornel JH, van der Zant FM, Knol RJ. Diagnostic and therapeutic usefulness of coronary computed tomography angiography in outclinic patients referred for chest pain. Am J Cardiol. 2015;116:30-36.

36. Stehli J, Fuchs TA, Bull S, et al. Accuracy of coronary CT angiography using a submillisievert fraction of radiation exposure: comparison with invasive coronary angiography. J Am Coll Cardiol. 2014;64:772-780.

37. Jerome SD, Tilkemeier PL, Farrell MB, Shaw LJ. Nationwide laboratory adherence to myocardial perfusion imaging radiation dose reduction practices: a report from the Intersocietal Accreditation Commission Data Repository. JACC Cardiovasc Imaging. 2015:8:1170-1176.

38. Mercuri M, Pascual TN, Mahmarian JJ, et al. INCAPS investigators group. Comparison of radiation doses and best-practice use for myocardial perfusion imaging in US and non-US laboratories: findings from the IAEA (internationa atomic Energy agency) nuclear cardiology protocols study. JAMA Intern Med. 2016;176:266-269.

39. Einstein AJ, Blankstein R, Andrews H, et al. Comparison of image quality myocardial perfusion, and left ventricular function between standard imaging and single-injection ultra-low-dose imaging using a high-efficiency SPECT camera: the MILLISIEVERT study. J Nucl Med. 2014;55:1430-1437.

40. Dey D, Slomka PJ, Berman DS. Achieving very-low-dose radiation exposure in cardiac computed tomography, single-photon emission computed tomography, and positron emission tomography. Circ Cardiovasc Imaging. 2014;7: 723-734.

41. Greenwood JP, Ripley DP, Berry C, et al. CE-MARC 2 Investigators, Effect of care guided by cardiovascular magnetic resonance, myocardial perfusion scintigraphy, or NICE Guidelines on subsequent unnecessary angiography rates: the CE-MARC 2 randomized clinical trial. JAMA. 2016;316:1051-1060.

42. Einstein AJ. Effects of radiation exposure from cardiac imaging: how good are the data? J Am Coll Cardiol. 2012;59:553-565.

43. Berrington de Gonzalez A, Kim KP, Smith-Bindman R, McAreavey D. Myocardial perfusion scans: projected population cancer risks from current levels of use in the United States. Circulation. 2010;122:2403-2410.

44. National Research Council (U.S.). Committee to Assess Health Risks from Exposure to Low Level of Ionizing Radiation. Health Risks from Exposure to Low Levels of Ionizing Radiation: BEIR VII Phase 2. Washington, DC: National Academies Press; 2006.

45. Fazel R, Krumholz HM, Wang Y, et al. Exposure to low-dose ionizing radiation from medical imaging procedures. N Engl J Med. 2009;361:849-857.

46. Bongartz G, Golding SJ, Jurik AG, et al. European guidelines for multislice computed tomography. Available at: http://www.msct.eu/CT_Quality_Criteria. htm; 2004.

47. Huda W, Tipnis S, Sterzik A, Schoepf UJ. Computing effective dose in cardiac CT. Phys Med Biol. 2010;55:3675-3684.

48. The 2007 recommendations of the international commission on radiological protection: ICRP publication 103. Ann ICRP. 2007;37:1-332.

49. Martin CJ. The application of effective dose to medical exposures. Radiat Prot Dosim. 2008;128:1-4. 